\title{
Top quark electroweak couplings at future lepton colliders
}

\author{
Christoph Englert $^{\mathrm{a}}$, Michael Russell ${ }^{\mathrm{b}}$ \\ School of Physics and Astronomy, University of Glasgow, Glasgow G12 8QQ, UK
}

Received: 18 April 2017 / Accepted: 20 July 2017 / Published online: 10 August 2017

(C) The Author(s) 2017. This article is an open access publication

\begin{abstract}
We perform a comparative study of the reach of future $e^{+} e^{-}$collider options for the scale of non-resonant new physics effects in the top quark sector, phrased in the language of higher-dimensional operators. Our focus is on the electroweak top quark pair production process $e^{+} e^{-} \rightarrow$ $Z^{*} / \gamma \rightarrow t \bar{t}$, and we study benchmark scenarios at the ILC and CLIC. We find that both are able to constrain mass scales up to the few $\mathrm{TeV}$ range in the most sensitive cases, improving by orders of magnitude on the forecast capabilities of the LHC. We discuss the role played by observables such as forward-backward asymmetries, and making use of different beam polarisation settings, and highlight the possibility of lifting a degeneracy in the allowed parameter space by combining top observables with precision $Z$-pole measurements from LEP1.
\end{abstract}

\section{Introduction}

Studies of the couplings of the top quark form one of the cornerstones of the analysis program pursued at the LHC, where top quark related processes, most notably $t \bar{t}$ production, are ubiquitous. Whilst improving the precision of Standard Model predictions is important in its own right, a key motivation for the detailed study of the top quark is to understand its potential role in the physics behind electroweak symmetry breaking, which has yet to be elucidated. As the only fermion with a Yukawa coupling $y_{t} \simeq 1$, the top is a pertinent place to look for the signatures of new physics at the $\mathrm{TeV}$ scale. Motivated scenarios range from compositeness theories (e.g. [1-3]) all the way to supersymmetry (see [4] for a review).

If the new physics that couples to the top quark is heavy and/or weakly coupled, an appropriate formulation of the effects of new physics is to view the SM as an effective the-

\footnotetext{
a e-mail: christoph.englert@ glasgow.ac.uk

be-mail: m.russell.2@ research.gla.ac.uk
}

ory, where the non-standard couplings are parameterised by operators of dimension $D>4$. The leading effects for collider observables typically enter at $D=6$,

$\mathcal{L}_{\text {eff }}=\mathcal{L}_{\mathrm{SM}}+\sum_{i} \frac{c_{i}^{(6)} \mathcal{O}_{i}^{(6)}}{\Lambda^{2}}+\cdots$

It is also typically justified to truncate the EFT expansion ${ }^{1}$ at this order. Global fits of top quark couplings to data from ATLAS and CMS are already well established (see e.g. Refs. [6-12] for a representative sample as well as [13] for a discussion of electroweak precision effects), and a similar effort is well under way in Higgs physics, where the associated limits on Wilson coefficients are typically statistics limited.

Still, despite the impressive statistical sample of top quark data that enters these fits, the subsequent direct bounds on the operator coefficients, and, by extension, the scale of new physics that would generate those operators, are rather weak [7]. There are few top quark measurements at the LHC that can be considered "precision observables" (helicity fractions in top decays are an exception [14]). By dimensional analysis, the strength of the interference of these operators with energy $\sqrt{s}$ typically scales as $s / \Lambda^{2}$, and the areas of phase space that are most sensitive are plagued by correlated experimental and theoretical systematics. Moreover, the associated weak limits translate into values of $\Lambda$ that are probed by the high energy bins of the measurement, bringing into question the validity of the truncated EFT description [5] and care needs to be taken when combining measurements of different exclusive energy ranges of a binned distribution [15]. Inclusive cross-sections, being typically dominated by the threshold region $\sqrt{s} \sim(2) m_{t}$, are under more theoretical control, but bring far less sensitivity.

\footnotetext{
${ }^{1}$ For counterexamples to this statement, see for example Ref. [5] and references therein.
} 
Lepton colliders are not vulnerable to either of these problems. Firstly, there is excellent control over the hard scale of the interaction $\sqrt{s}$, so one can always ensure that the limits on the $D=6$ operators are consistent with a wellbehaved EFT expansion. ${ }^{2}$ Secondly, the theoretical uncertainties from Standard Model calculations are much smaller: there are no PDFs, and the current state of the art precision for $t \bar{t}$ production is $\mathrm{N}^{3} \mathrm{LO}$ QCD at fixed-order [16], and NNLO+NNLL including threshold resummation, which bring SM scale uncertainty variation bands to the percent level [17].

The physics case for a $e^{+} e^{-}$collider is by now well established. The principal motivation is to perform a detailed precision study of the couplings of the Higgs boson in the much cleaner environment that a lepton collider affords, which will bring Higgs coupling measurements to an accuracy that will not be challenged by the LHC, even after it collects $3 \mathrm{ab}^{-1}$ of data $[18,19]$.

The electroweak couplings of the top quark are also clearly within the remit of such a collider. Currently, the only handle on top quark electroweak couplings from the LHC is through the associated production $p p \rightarrow t \bar{t} V$ where $V \in\{Z, W, \gamma\}$. Whilst measurements of these processes are now approaching the $5 \sigma$ level, the pull that they have on a global fit is small [7]. Measurements of electroweak single top production bring stronger bounds, but they are sensitive to a smaller subset of operators.

At a lepton collider, on the other hand, the process $e^{+} e^{-} \rightarrow Z^{*} / \gamma \rightarrow t \bar{t}$ is extremely sensitive to top electroweak couplings. While the overall rate is more modest than at the LHC due to the parametric $\alpha_{E W} / \alpha_{s}$ and $s$-channel suppression, the process is essentially background free, and it would constitute the first true precision probe of the electroweak sector of the top quark, and open up a new avenue for top quark couplings, complementary to the well-studied top QCD interactions.

Several studies of the prospects for improvement of top measurements at future colliders have already been undertaken (see for example Refs. [20-25]), in particular for the proposed International Linear Collider (ILC), but none have explicitly quantified the gain in the constraints on the top electroweak sector of the SMEFT, nor provided a comparative study of different collider options. The purpose of this paper is to provide such a study.

In Sect. 2 we discuss the $e^{+} e^{-} \rightarrow Z^{*} / \gamma \rightarrow t \bar{t}$ process in the Standard Model EFT, and the $D=6$ operators that generate interference with the Standard Model. To motivate the study, in Sect. 4 we discuss the present status of constraints

\footnotetext{
${ }^{2}$ Consistently improving the perturbative precision within the dimension 6 framework, however, makes the truncation of the perturbative series necessary as corrections to $(D=6)^{2}$ operators will typically require unaccounted $D=8$ counterterms.
}

on top electroweak couplings, and discuss projections for the lifetime of the LHC. The rest of the paper is devoted to lepton collider projections. In Sect. 5 we discuss ILC constraints, based on the H-20 running scenario. In Sect. 6, we focus on prospects from CLIC, comparing the potential of the two future collider projections. In Sect. 7 we analyse the complementarity between the bounds derived from $t \bar{t}$ measurements at a future collider and $Z$-pole measurements, before summarising in Sect. 8.

\section{Top electroweak couplings}

In the Standard Model, the electroweak $t \bar{t} Z$ coupling is given by the vector-axial-vector coupling

$\mathcal{L}_{\mathrm{ttZ}}=e \bar{t}\left[\gamma^{\mu}\left(v_{t}-\gamma_{5} a_{t}\right)\right] t Z_{\mu}$

where

$$
\begin{aligned}
& v_{t}=\frac{T_{t}^{3}-2 Q_{t} \sin ^{2} \theta_{W}}{2 \sin \theta_{W} \cos \theta_{W}} \simeq 0.24, \\
& a_{t}=\frac{T_{t}^{3}}{2 \sin \theta_{W} \cos \theta_{W}} \simeq 0.60 .
\end{aligned}
$$

To capture effects beyond the SM in this Lagrangian there are two approaches: one can write down anomalous couplings for the $t \bar{t} Z$ vertex, such that $\mathcal{L}_{\text {ttZ }}$ receives a term

$$
\begin{aligned}
\Delta \mathcal{L}_{\mathrm{ttZ}}= & e \bar{t}\left[\left(\gamma^{\mu}\left(C_{1 V}+\gamma_{5} C_{1 A}\right)\right.\right. \\
& \left.+\frac{i \sigma^{\mu \nu} q_{v}}{2 M_{Z}}\left(C_{2 V}+\gamma_{5} C_{2 A}\right)\right] t Z_{\mu}
\end{aligned}
$$

where $q=p_{t}-p_{\bar{t}}$. While this has the advantage of elucidating the various spin structures that can impact the $t \bar{t} Z$ vertex, it has the drawback that it does not allow for a simple power counting of which anomalous couplings would have the strongest effect. For example, the coefficient $C_{2 A}$ is zero in the Standard Model, so that any corrections to it come solely from new physics contributions, which should be smaller than couplings that have SM interference.

To augment this description, one can instead supplement Eq. (II.1) with higher-dimensional operators. At leading order in the SMEFT, the list of operators that generate modifications to the $t \bar{t} Z$ vertex is, expressed in the basis and notation of Ref. [26]:

$$
\begin{aligned}
\mathcal{O}_{u W} & =\left(\bar{Q} \sigma^{\mu v} u\right) \tau^{I} \tilde{\varphi} W_{\mu \nu}^{I}, \\
\mathcal{O}_{u B} & =\left(\bar{Q} \sigma^{\mu v} u\right) \tilde{\varphi} B_{\mu \nu}, \\
\mathcal{O}_{\varphi q}^{(3)} & =\left(\varphi^{\dagger} i \overleftrightarrow{D_{\mu}^{I}} \varphi\right)\left(\bar{Q} \tau^{I} \gamma^{\mu} Q\right), \\
\mathcal{O}_{\varphi q}^{(1)} & =\left(\varphi^{\dagger} i \overleftrightarrow{D_{\mu}} \varphi\right)\left(\bar{Q} \gamma^{\mu} Q\right),
\end{aligned}
$$




$$
\mathcal{O}_{\varphi u}=\left(\varphi^{\dagger} i \overleftrightarrow{D_{\mu}} \varphi\right)\left(\bar{u} \gamma^{\mu} u\right)
$$

The dictionary between the $D=6$ operators of Eq. (II.4) and the anomalous couplings of Eq. (II.3) is

$$
\begin{aligned}
C_{1 V} & =\frac{v^{2}}{\Lambda^{2}} \Re\left[c_{\varphi q}^{(3)}-c_{\varphi q}^{(1)}-c_{\varphi u}\right]^{33}, \\
C_{1 A} & =\frac{v^{2}}{\Lambda^{2}} \Re\left[c_{\varphi q}^{(3)}-c_{\varphi q}^{(1)}+c_{\varphi u}\right]^{33}, \\
C_{2 V} & =\sqrt{2} \frac{v^{2}}{\Lambda^{2}} \Re\left[\cos \theta_{W} c_{u W}-\sin \theta_{W} c_{u B}\right]^{33}, \\
C_{2 A} & =\sqrt{2} \frac{v^{2}}{\Lambda^{2}} \Im\left[\cos \theta_{W} c_{u W}+\sin \theta_{W} c_{u B}\right]^{33},
\end{aligned}
$$

where the superscript 33 denotes that we are considering the third generation only in the fermion bilinears of Eq. (II.4). Since $c_{\varphi q}^{(3)}$ and $c_{\varphi q}^{(1)}$ only appear with an overall opposite sign, we can only constrain the operator $\mathcal{O}_{\varphi q}^{(3)}-\mathcal{O}_{\varphi q}^{(1)} \equiv \mathcal{O}_{\varphi q}$ from $t \bar{t} Z$ couplings. We will discuss a method for bounding the two operators independently later in the paper.

$C_{2 A}$ is generated by a $\mathcal{C P}$-odd combination of operators, therefore it does not interfere with SM amplitudes and so its effects are expected to be smaller. Since in this study we are more interested in the absolute mass scales of these operators, we set all Wilson coefficients to be real, ${ }^{3}$ however, we note that $\mathcal{C P}$-sensitive observables such as angular distributions can also distinguish the $\mathcal{C P}$ character of the Wilson coefficients. We also assume that the new physics solely impacts the $t \bar{t} Z$ vertex, so we do not consider operators which modify the $Z e \bar{e}$ vertex, nor four-fermion operators which can contribute to the $q \bar{q} \rightarrow t \bar{t}$ or $e^{+} e^{-} \rightarrow t \bar{t}$ processes, though we note that including these would in general weaken our bounds (see e.g. Refs. [7,11] for constraints on the former and Ref. [13] for the latter).

\section{Analysis setup}

We implemented the operators of Eq. (II.4) in a FEYNRULES [28] model file, which was exported via UFO [29] to MADGRAPH [30], in order to generate parton-level events. For the analytic results discussed later, the model file was exported to FEYNARTS/FORMCALC [31,32], which we used to calculate the full dependence of each observable used on the operators of Eq. (II.4). To generate lepton collider constraints, we minimise the $\chi^{2}$ between the full 5D analytic expression and SM pseudodata using IMINUIT, a Python/C++ implementation of the MINUIT [33] algorithm. For hadron collider constraints, we approximate the full 5D dependence

\footnotetext{
3 This is a well-motivated assumption considering that $\mathcal{C P}$-odd components of the operators we consider are very tightly constrained by EDM experiments[27].
}

of the cross-section on the operators using PROFESSOR; an interpolation-based method described in Ref. [34]. We crosscheck our analytic results against Monte Carlo scans using a UFO implementation of the full basis of Ref. [26] and find good agreement.

\section{Hadron collider constraints}

To appreciate the impact of the operators of Eq. (II.4), in Fig. 1 we plot the ratio of the full $t \bar{t} Z$ cross-section with each operator switched on individually, to the NLO SM prediction, taken from Ref. [35]. For ease of interpretation, we split up the cross-section into the contribution from the interference term and the quadratic term. We see firstly that the operators $\mathcal{O}_{u W}$ and $\mathcal{O}_{u B}$ have the strongest impact on the total crosssection, but this comes purely from the squared term (this was also noted in Ref. [8]). The remaining operators have a milder effect on the cross-section, but their interference term dominates. We also see that the operators $\mathcal{O}_{\varphi q}^{(3)}$ and $\mathcal{O}_{\varphi q}^{(1)}$ contribute the same dependence but with an opposite sign, as discussed in Sect. 2, therefore we can only bound the linear combination $\mathcal{O}_{\varphi q}$.

The LHC bounds on the coefficients of these operators from $8 \mathrm{TeV} t \bar{t} Z$ production cross-sections have been presented in Ref. [7]. The current constraints are weak. Since then, ATLAS and CMS have presented measurements using $13 \mathrm{TeV}$ collision data, with measured values $0.9 \pm 0.3 \mathrm{pb}$ [36] and $0.7 \pm 0.21 \mathrm{pb}$ [37], respectively. The constraints on the operators using these two measurements are shown in Fig. 2, where the coefficients are normalised to the 'bar' notation $\bar{c}_{i}$ $=c_{i} v^{2} / \Lambda^{2}$, and the operators are switched on individually.

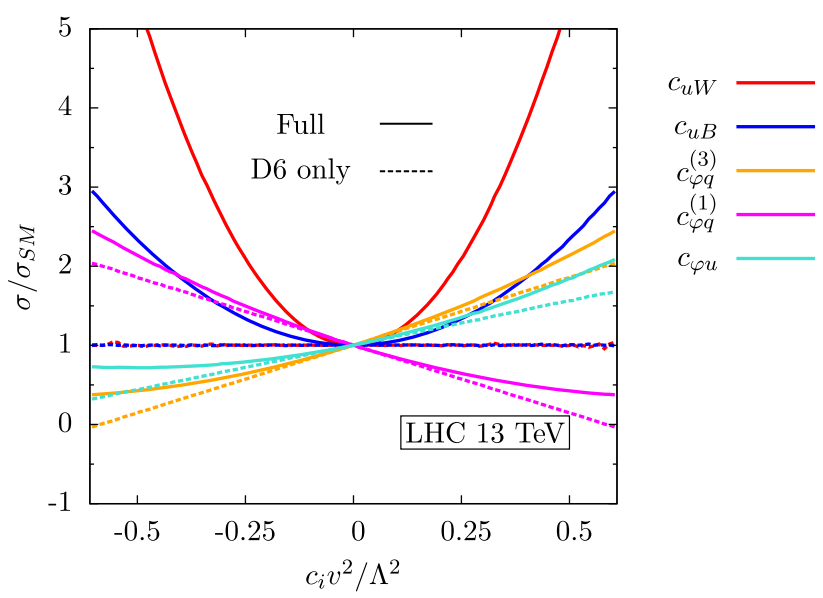

Fig. 1 Ratio of the full SM $p p \rightarrow t \bar{t} Z$ cross-section with the operators of Eq. (II.4) switched on individually to the NLO Standard Model estimate. The dashed lines show the contribution from the interference term, and the solid lines show the full dependence 


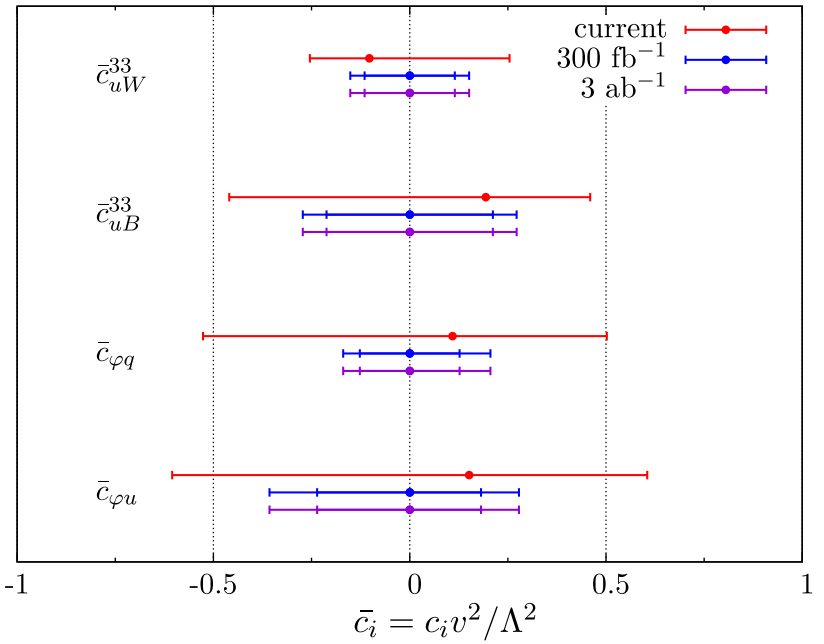

Fig. 2 Individual $95 \%$ confidence intervals on the coefficients of the operators of Eq. (II.4) using the current $13 \mathrm{TeV}$ measurements (red bars). Also shown are the projected constraints using $300 \mathrm{fb}^{-1}$ (blue) and $3 \mathrm{ab}^{-1}$ (purple) of SM pseudodata. For the latter two cases, the inner bars show the improvement when theory uncertainties are reduced to $1 \%$

We see that the current constraints are still quite weak, mainly due to the large $(\sim 30 \%)$ experimental uncertainties. These measurements are currently statistics dominated, so it is instructive to ask what the expected improvement is over the lifetime of the LHC. Using a constant systematic uncertainty of $10 \%$ based on the current estimate, we also plot in Fig. 2 the constraints using $300 \mathrm{fb}^{-1}$ and $3 \mathrm{ab}^{-1}$ of SM pseudodata. We see that there will be an improvement by factors of 1.5 to 2 by the end of Run III, but after this the measurement is saturated by systematics.

To highlight the benefits of improving the theory description in tandem, we also show in Fig. 2 the projected constraints if theory uncertainties are improved to $1 \%$ from the current $\mathcal{O}(10 \%)$ precision, which does not seem unreasonable over the timescales we are considering. We see again that there will be no subsequent improvement after $300 \mathrm{fb}^{-1}$ unless experimental systematics are reduced.

Finally, it should be noted that as more data becomes available, it may be possible to measure $t \bar{t} Z$ cross-sections differentially in final state quantities. Since cuts on the final state phase space can enhance sensitivity to the region where naïve power counting says $D=6$ operators become more important, differential distributions could substantially improve the fit prospects, as has already been demonstrated for $t \bar{t}$ production. [7,38].

To illustrate this, in Fig. 3 we plot the distributions for the $Z$ boson transverse momentum and top pair transverse momentum, both for the SM only case and with each operator switched on to a value of $\bar{c}_{i} \simeq 0.3$; approximately the maximum allowed by current constraints in Fig. 2. We see that extra enhancement in the tail is visible for the field strength tensor operators $\mathcal{O}_{u W}$ and $\mathcal{O}_{u B}$, due to the extra momentum dependence in the numerator from the field strength tensor. For the $\varphi$-type operators, since the interference is solely proportional to $\varphi^{\dagger} \varphi \rightarrow v^{2} / \Lambda^{2}$, there is no extra enhancement at high $p_{T}$.

We do not estimate the improvement of the fit by taking these distributions into account, since this would require proper estimates of experimental systematics and tracking the nontrivial correlations between the kinematic quantities in the massive 3-body final state. Here, we merely comment that it may be an avenue worth pursuing as more data becomes available.

\section{ILC constraints}

Going beyond the LHC, currently, the most mature proposal is for a linear $e^{+} e^{-}$collider with a centre of mass energy ranging from $250 \mathrm{GeV}$ to up to $1 \mathrm{TeV}$. There are several scenarios for integrated luminosity and CM energy combinations. The most-studied is the so-called $\mathrm{H}-20$ option, which involves running at $500 \mathrm{GeV}$ for $500 \mathrm{fb}^{-1}$ of data, followed by $200 \mathrm{fb}^{-1}$ of data at the $t \bar{t}$ threshold to perform detailed measurements of the top quark mass, and $300 \mathrm{fb}^{-1}$ of data at $\sqrt{s}=250 \mathrm{GeV}$ to maximise the machine's Higgs potential with high precision. After a luminosity upgrade, a further 3.5 $\mathrm{ab}^{-1}$ is gathered at $\sqrt{s}=500 \mathrm{GeV}$, followed by another $\sqrt{s}$ $=250 \mathrm{GeV}$ run at $1.5 \mathrm{ab}^{-1}$. Since we are most interested in the ILC mass reach for new physics, in this study we focus on the $500 \mathrm{GeV}$ ILC running. ${ }^{4}$

An important parameter for lepton colliders is the energy spread of electron and positron beams (see e.g. [39]). In order to estimate the effect on our results, we use the results of [39] to calculate the expected change in the cross- section by including the effects of initial state radiation, beam spread and beamstrahlung. We find that, for the typical beam profile, the associated uncertainty is not a limiting factor and we neglect these effects in the following.

\subsection{The $t \bar{t}$ total cross-section}

Top pair production has a more modest rate here than at a hadron collider. The state-of-the-art Standard Model calculations for (unpolarised) $e^{+} e^{-} \rightarrow t \bar{t}$ production are at $\mathrm{N}^{3} \mathrm{LO} Q C D$ in the narrow-width approximation [16,17], and at NLO QCD including off-shell effects (which have been demonstrated to be important [40]) and at NLO EW [41] (with partial NNLO results in Ref. [42]) and predict a cross-

\footnotetext{
${ }^{4}$ In principle, the constraints could be improved by also adding results from $\sqrt{s}=350 \mathrm{GeV}$, however, this requires more precise theory modelling to understand the impact of $D=6$ operators on threshold effects than our leading-order $+K$-factor analsysis.
} 


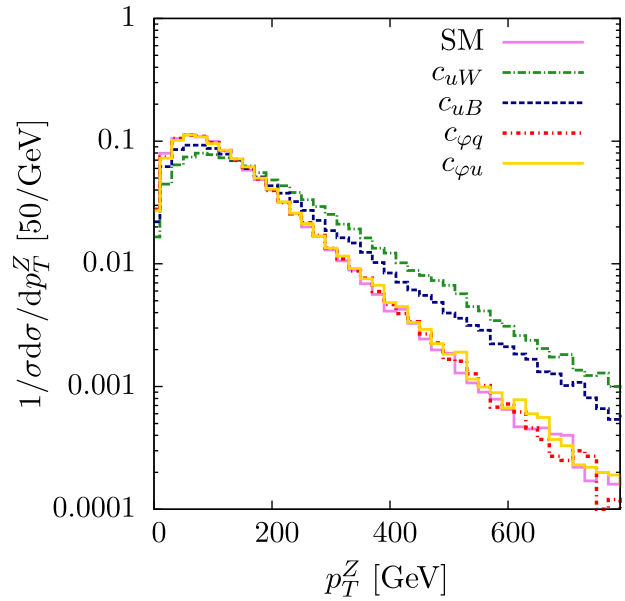

Fig. 3 Kinematic distributions in $p p \rightarrow t \bar{t} Z$ production at $13 \mathrm{TeV}$ for the SM prediction and for the operators of Eq. (II.4) switched on to their maximum value allowed by current data. Left The $Z$ boson transverse momentum spectrum. Right $p_{T}^{t \bar{t}}=p_{T}^{t}-p_{T}^{\bar{t}}$ spectrum. All distributions

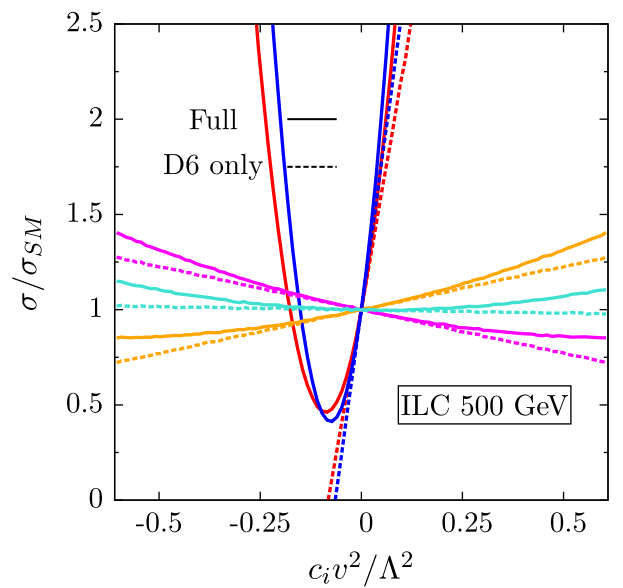

(a)

Fig. 4 a Ratio of the full SM $e^{+} e^{-}$to $t \bar{t}$ cross-section at $\sqrt{s}=500$ $\mathrm{GeV}$ with the operators of Eq. (II.4) switched on individually to the NLO Standard Model estimate. The dashed lines show the contribution

section $\sigma \simeq 0.57 \mathrm{pb}$. The conventional scale variation gives a QCD uncertainty at the per-mille level. While this rate is more than a factor of a thousand smaller than at the 13 $\mathrm{TeV}$ LHC, the process is essentially background free. Thus, after even $500 \mathrm{fb}^{-1}$ of data the statistical uncertainty will be approximately $0.2 \%$, and so completely subdominant to the systematics.

We can thus repeat the exercise of extracting the bounds on the coefficients of the operators of Eq. (II.4) using SM pseudodata. As a guide for the expected numerical constraints, we also plot the ratio of the total cross-section in the presence of the operators to the SM prediction, this time using the

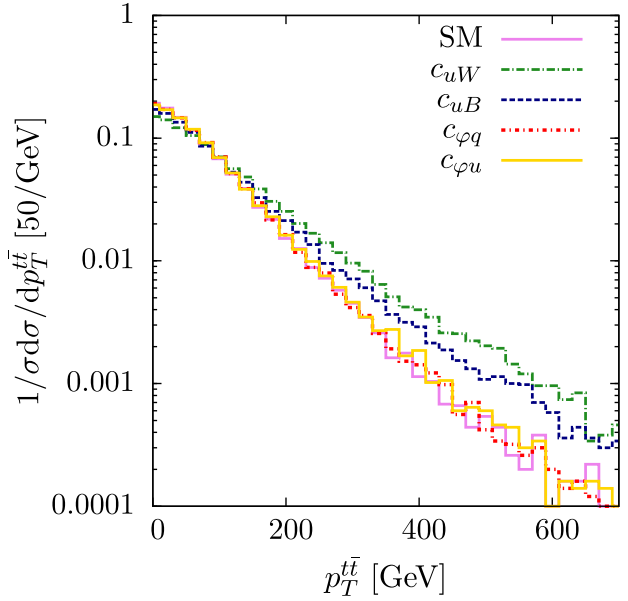

are normalised to the total cross-section. Shape differences can be seen in the tails for the operators $\mathcal{O}_{u W}$ and $\mathcal{O}_{u B}$, showing that differential distributions provide complementary information to overall rates

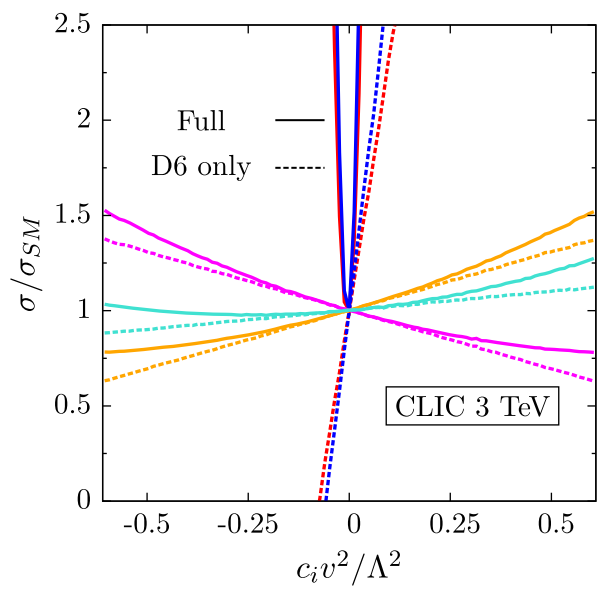

(b)

from the interference term, and the solid lines show the full dependence. The operator colour coding is the same as Fig. 1. b Likewise for CLIC running at $\sqrt{s}=3 \mathrm{TeV}$

total (unpolarised) cross-section at the $500 \mathrm{GeV}$ ILC. This is shown on the left of Fig. 4.

We see again that the operators $\mathcal{O}_{u W}$ and $\mathcal{O}_{u B}$ are the strongest, however, unlike the case of $t \bar{t} Z$ production the interference term dominates at small $c_{i} / \Lambda^{2}$. The result of this is that there is a cancellation between the interference and quadratic terms at approximately $c_{i} / \Lambda^{2} \simeq-3 \mathrm{TeV}^{-2}$, leading to a SM-like cross-section and a second, degenerate minimum in the $\chi^{2}$. The constraints obtained from a one-ata-time fit of these operators to the SM pseudodata is shown in the red bars on the right of Fig. 6 .

The operators $\mathcal{O}_{u W}$ and $\mathcal{O}_{u B}$ are very tightly constrained, due to their much stronger impact on the cross-section stem- 


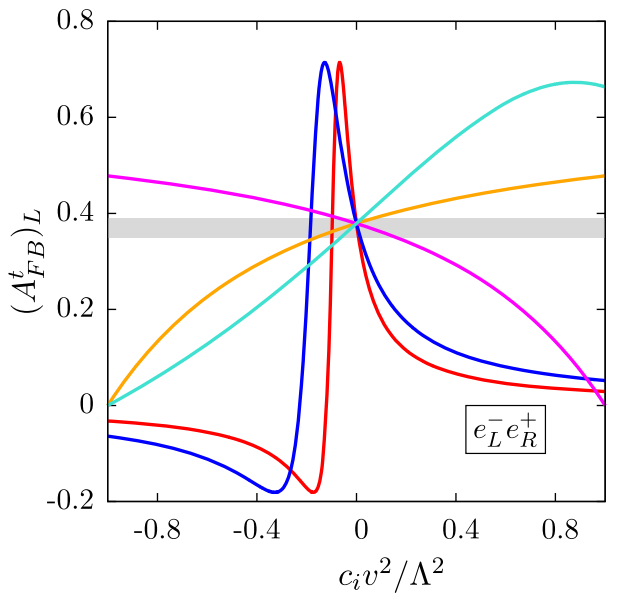

Fig. 5 Full dependence of the $t \bar{t}$ forward-backward asymmetry of Eq. (V.1) on the operators of Eq. (II.4) for left-handed polarised electrons (left) and right-handed polarised electrons (right) at $\sqrt{s}=500$

ming from the extra momentum dependence flowing through the vertex. The $\varphi$-type operators are more weakly constrained, but on the whole the constraints are typically 10-100 times stronger than for the LHC $t \bar{t} Z$ production projections in Sect. 4, which is unsurprising giving the difference in precision.

Individual constraints are less useful in practice, however. Firstly, in a plausible UV scenario that would generate these operators one would typically expect more than one to be generated at once, so that one-at-a-time constraints cannot be straightforwardly linked to a specific 'top-down' model. Secondly, there can in general be cancellations between different operators for a given observable that can yield spurious local minima and disrupt the fit. This would not be visible in the individual constraints, and so would obscure degeneracies in the operator set that could be broken by considering different observables. Therefore, we also consider constraints where we marginalise over the remaining three coefficients in the fit. These are shown in the blue bars on the right of Fig. $6 .^{5}$

We see that, with the exception of $\mathcal{O}_{u W}$ and $\mathcal{O}_{u B}$, marginalising over the full operator set wipes out the constraints. This is because even for large values of coefficients, the pull that a particular operator has on the cross-section can easily be cancelled by another operator. We can conclude that, despite the impressive precision that can be achieved in extracting the cross-section, it has limited use in constraining new physics in a simultaneous global fit of several operators. It is worthwhile to make use of other measurements.

\footnotetext{
5 Note, however, that a full marginalisation will be overly conservative when confronting a concrete UV model.
}

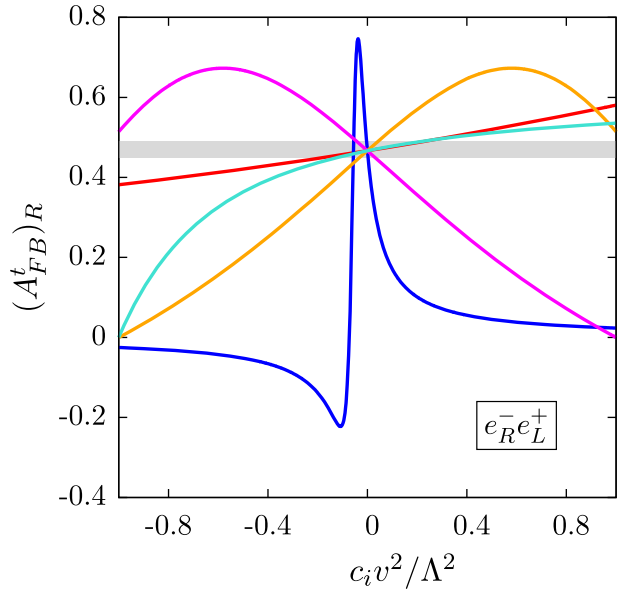

$\mathrm{GeV}$, the operator colour coding is the same as Fig. 1. We also show a $5 \%$ uncertainty band around the SM prediction, to estimate the expected constraints

\subsection{Polarised beams}

One of the principal strengths of lepton colliders is that the polarisation of the incoming beams can be finely controlled, so that the relative contributions between different subprocesses to a given final state can be tuned. Moreover, because the dependence of top observables on the operators of Eq. (II.4) depends strongly on the initial state polarisation, varying the settings increases the number of independent measurements that can be used to place bounds in a global fit.

To emphasise this point, we study the forward-backward asymmetry, defined as

$A_{F B}^{t}=\frac{N\left(\cos \theta_{t}>0\right)-N\left(\cos \theta_{t}<0\right)}{N\left(\cos \theta_{t}>0\right)+N\left(\cos \theta_{t}<0\right)}$

where $\theta_{t}$ is the polar angle between the top quark and the incoming electron, for three incoming beam polarisation settings: unpolarised beams, denoted $\left(A_{F B}^{t}\right)_{U}$; a fully lefthanded initial polarised electron beam and fully right-handed polarised positron beam, denoted $\left(A_{F B}^{t}\right)_{L}$; and vice versa, denoted $\left(A_{F B}^{t}\right)_{R}$. The SM predictions for these settings at tree level are $\left\{\left(A_{F B}^{t}\right)_{U},\left(A_{F B}^{t}\right)_{L},\left(A_{F B}^{t}\right)_{R}\right\} \simeq\{0.40,0.37,0.47\}$, which agree well with the full NNLO QCD estimates $[43,44]$. The dependence of these asymmetries on the operators of Eq. (II.4) is shown in Fig. 5.

We see that the dependence on the operators distinctively depends on the initial state polarisations. For the $\left(A_{F B}^{t}\right)_{L}$ case, we again see the large interference-square cancellation in the gauge-type operators $\mathcal{O}_{u W}$ and $\mathcal{O}_{u B}$. For the righthanded case the impact of $\mathcal{O}_{u W}$ is much milder. For both cases we see that the operators $\mathcal{O}_{\varphi q}^{(3)}$ and $\mathcal{O}_{\varphi q}^{(1)}$ pull the prediction in opposite directions. Most encouragingly, we see that 


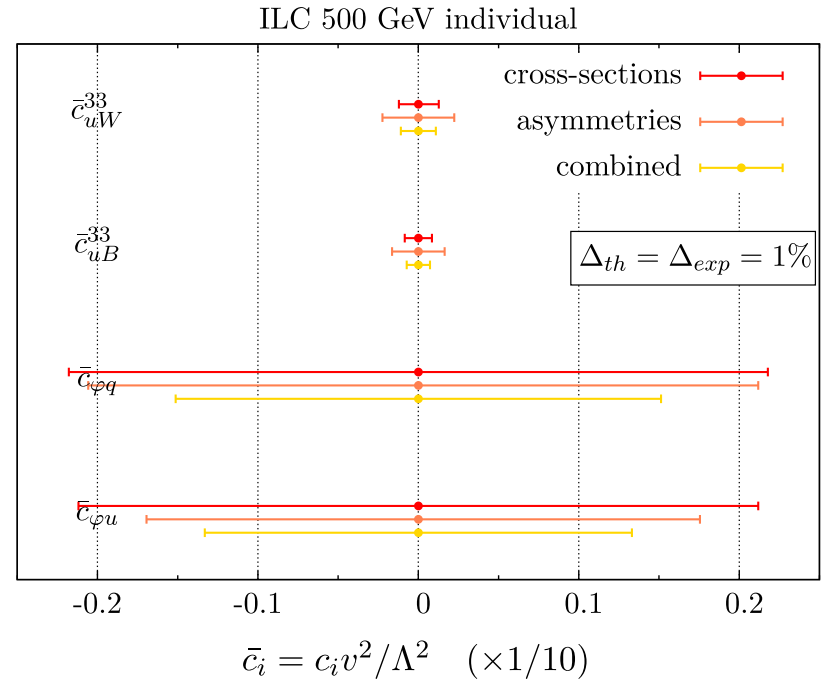

(a)

Fig. $695 \%$ confidence ranges for the operators we consider here, from the $500 \mathrm{GeV}$ ILC, assuming $1 \%$ theoretical and experimental uncertainties, by fitting to cross-sections, asymmetries, and the combination, with

the departure from the SM prediction is now much stronger for the $\varphi$-type operators than the total cross-section, which should lead to a sizeable improvement in the final constraints.

To generate these constraints, we consider a global fit of the four operators to six observables:

$\left\{\left(A_{F B}^{t}\right)_{U},\left(A_{F B}^{t}\right)_{L},\left(A_{F B}^{t}\right)_{R},\left(\sigma_{\text {tot }}^{t \bar{t}}\right)_{U},\left(\sigma_{\text {tot }}^{t \bar{t}}\right)_{L},\left(\sigma_{\text {tot }}^{t \bar{t}}\right)_{R}\right\}$.

In extracting the constraints, we consider the more realistic ILC polarisation capabilities $\mathcal{P}_{e^{-}}= \pm 0.8, \mathcal{P}_{e^{+}}=\mp 0.3$, noting that the cross-section for arbitrary $e^{+} e^{-}$polarisations is related to the fully polarised one by $[45,46]$

$$
\begin{aligned}
\sigma_{\mathcal{P}_{e^{-}} \mathcal{P}_{e^{+}}=} & \frac{1}{4}\left\{\left(1+\mathcal{P}_{e^{-}}\right)\left(1-\mathcal{P}_{e^{+}}\right) \sigma_{\mathrm{RL}}\right. \\
& \left.+\left(1-\mathcal{P}_{e^{-}}\right)\left(1+\mathcal{P}_{e^{+}}\right) \sigma_{\mathrm{LR}}\right\},
\end{aligned}
$$

where $\sigma_{\mathrm{RL}}$ is the cross-section for fully right-handed polarised electrons and fully left-handed polarised positrons and $\sigma_{\mathrm{RL}}$ is vice versa (the $\sigma_{\mathrm{RR}}$ and $\sigma_{\mathrm{LL}}$ components vanish for $p$-wave annihilation into spin-1 bosons). Performing a $\chi^{2}$ fit of the full analytic expression for each observable, using SM pseudodata with $1 \%$ experimental error bars (based on studies in Refs. $[22,47]$ ) and SM theory uncertainties of $1 \%$ (based on the calculations of Refs. $[16,17,41,43,44])$ the individual and marginalised constraints on these operators are shown in Fig. 6.

At the level of individual operators, the constraints are not improved drastically by adding in asymmetry information. For the global fit, however, the constraints lead to much

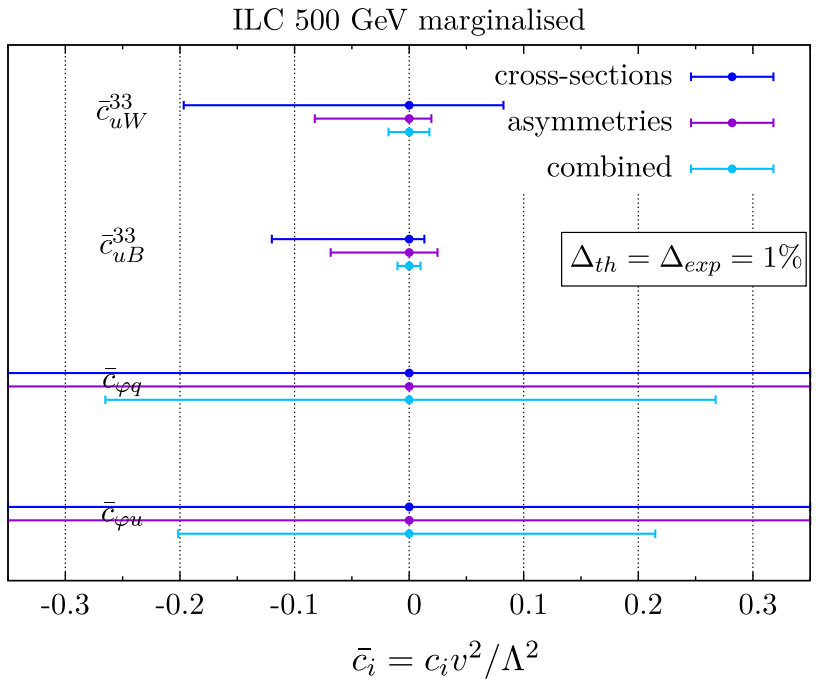

(b)

each operator considered individually (a) or in a 5D fit (b). To display both on the same plot, we scale the individual constraints up by a factor of 10 , so that the bottom axis is actually $\bar{c}_{i} / 10$

stronger bounds than for fitting to cross-sections [although the marginalisation typically weakens the overall constraints by a factor $\mathcal{O}(10)]$.

We see that the constraints are again much stronger for the field strength operators $\mathcal{O}_{u W}$ and $\mathcal{O}_{u B}$, where the constraints are at the $\left|\bar{c}_{i}\right| \lesssim 10^{-4}$ level for the individual constraints and $\left|\bar{c}_{i}\right| \lesssim 10^{-2}$ for the marginalised case, corresponding to a mass reach of $\Lambda \gtrsim 10 \mathrm{TeV}$ and $\Lambda \gtrsim 2.16 \mathrm{TeV}$, respectively, assuming $c_{i} \simeq 1$. The weakest constraints are on the operators $\mathcal{O}_{\varphi q}^{(3)}\left(\mathcal{O}_{\varphi q}^{(1)}\right)$, which translate into bounds on $\Lambda$ of roughly $700 \mathrm{GeV}$.

While it is encouraging that the bounds are consistent with an EFT formulation, in the sense that $\Lambda \gg \sqrt{s}$, the ILC mass reach for the scale of new physics that would generate these operators is still low. We note, however, that these bounds are on the conservative side, since other observables such as oblique parameters and LEP asymmetries contribute complementary information that will in general tighten them. To keep this fit self-contained, we postpone this discussion until Sect. 7.

\section{CLIC constraints}

The Compact Linear Collider (CLIC) project [48,49], with its larger maximum centre-of-mass energy $\sqrt{s}=3 \mathrm{TeV}$, will be in a stronger position to discover the effects of some higherdimensional operators, whose effects naïvely scale with the CM energy as $s / \Lambda^{2}$. There are two main running scenarios, but both envisage total integrated luminosities of $500 \mathrm{fb}^{-1}$ at $\sqrt{s}=500 \mathrm{GeV}, 1.5 \mathrm{ab}^{-1}$ at 1.4 or $1.5 \mathrm{TeV}$, and $2 \mathrm{ab}^{-1}$ at 3 


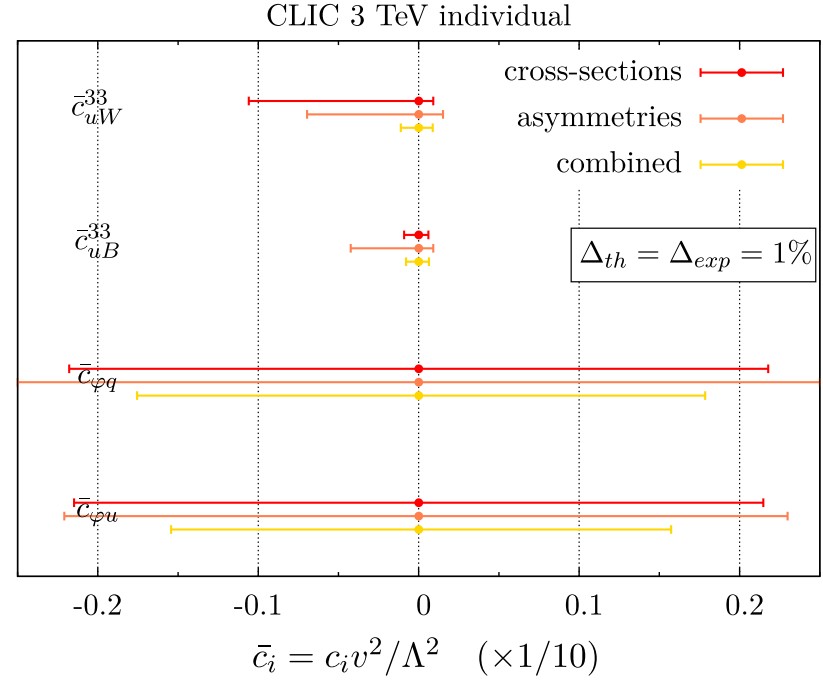

(a)

Fig. $795 \%$ confidence ranges for the operators we consider here, from CLIC running at $\sqrt{s}=3 \mathrm{TeV}$, assuming $1 \%$ theoretical and experimental uncertainties, by fitting to cross-sections, asymmetries, and the combi-

TeV. Again, we focus on the highest energy setting $\sqrt{s}=3$ $\mathrm{TeV}$, to maximise discovery potential for non-resonant new physics through $D=6$ operators.

Moving further away from the $t \bar{t}$ threshold, the total $e^{+} e^{-}$ $\rightarrow Z^{*} / \gamma \rightarrow t \bar{t}$ rate is smaller than at the ILC; at $\sqrt{s}=3 \mathrm{TeV}$ it is around $20 \mathrm{fb}$, which means for the total forecast integrated luminosity at this energy there will be a statistical uncertainty of $\simeq 0.5 \%$. A total experimental uncertainty of $1 \%$ may therefore be too optimistic an estimate once systematics are fully itemised. Nonetheless, for ease of comparison with the ILC figures, we take this as a baseline, and the corresponding constraints, using the same observables and beam settings, are shown in Fig. 7.

We see that, for the individual fit, CLIC constraints are of the same order of magnitude as the ILC ones. ${ }^{6}$ Although the direct sensitivity to the operators is enhanced, we see that as we move away from the $t \bar{t}$ threshold, the interference effect of the $\varphi$-type operators is much smaller. This is not the case for the operators $\mathcal{O}_{u W}$ and $\mathcal{O}_{u B}$, whose contributions stem mainly from the $(D=6)^{2}$ term, as seen on the right of Fig. 4 , which receives no suppression. Their individual constraints are close to the ILC values, indicating that energy scale is not the dominant factor driving these limits, but rather the theory and experimental uncertainties which saturate the sensitivity, which we do not vary.

For the more general marginalised fit, we see again that combining cross-section and asymmetry measurements will

\footnotetext{
6 This is in contrast to Higgs sector constraints from $e^{+} e^{-} \rightarrow h Z$, where the projected sensitivity is extremely dependent on the momentum flow through the vertex, leading to better overall CLIC constraints [50].
}

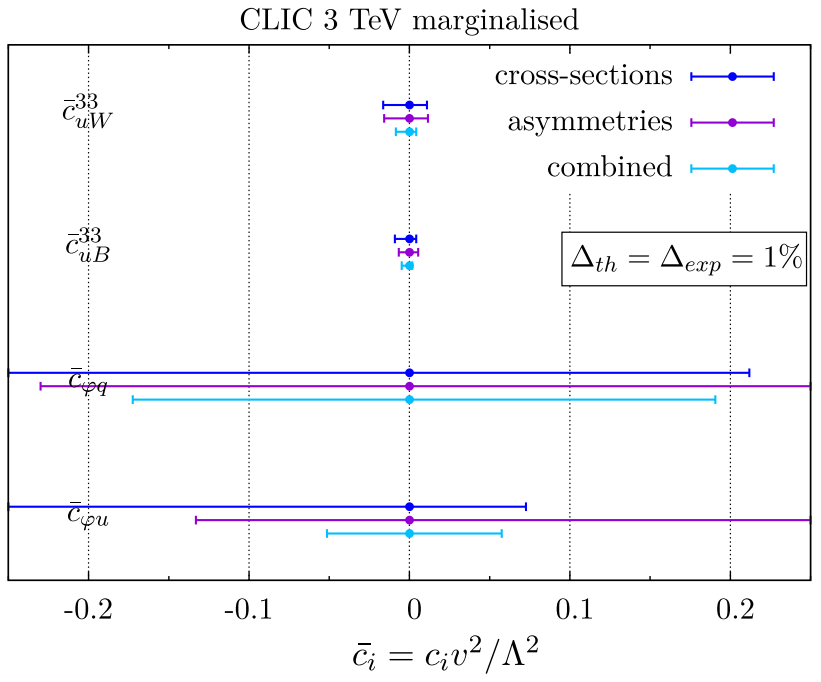

(b)

nation, with each operator considered individually (a) or in a 5D fit (b). To display both on the same plot, we scale the individual constraints up by a factor of 10 , so that the bottom axis is actually $\bar{c}_{i} / 10$

break blind directions in the fit, leading to much more powerful overall constraints. Unlike for the case of the ILC, however, care must be taken in interpreting these limits in terms of the mass scale of a particular UV model. The marginalised constraint $\left|\bar{c}_{\varphi u}\right| \lesssim 0.05$, for example, corresponds to a mass scale $\Lambda / \sqrt{c} \gtrsim 1.1 \mathrm{TeV}$, which is less than the energy scale probed in the interaction, so that the constraint can only be linked to a particular model if it is very weakly coupled: $g_{*} \ll 1$.

\section{Beyond $e^{+} e^{-} \rightarrow t \bar{t}$ : precision observables}

Obviously the direct constraints that we have focussed on in this work do not exist in a vacuum and the interplay of direct and indirect sensitivity plays an important part in ultimately obtaining the best constraints for a given model (see [51,52]). To put the expected direct constraints detailed above into perspective we analyse the impact of the considered operators on LEP precision observables. Note, that these $Z$ resonance observables are sensitive to a plethora of other new interactions and a direct comparison is not immediately straightforward [51]. Nonetheless, there is significant discriminative power that is worthwhile pointing out, which we will discuss in the following.

\subsection{Oblique corrections}

The $S, T, U$ parameters [54,55] (see also [56]) are standard observables that capture oblique deviations in the SM electroweak gauge sector from the SM paradigm $[57,58]$ through 


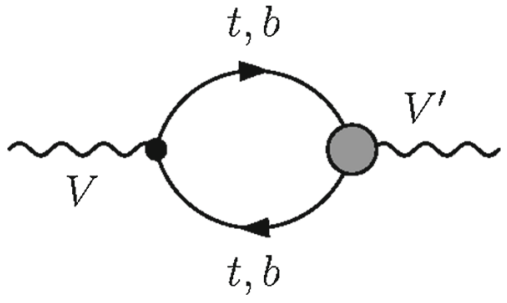

Fig. 8 Representative Feynman diagram contributing to the $S, T, U$ parameters at one-loop. The grey-shaded area marks a possible dimension six insertion while the black dot represents a SM vertex of the $V-V^{\prime}$ polarisation function, $V, V^{\prime}=W^{ \pm}, Z, \gamma$

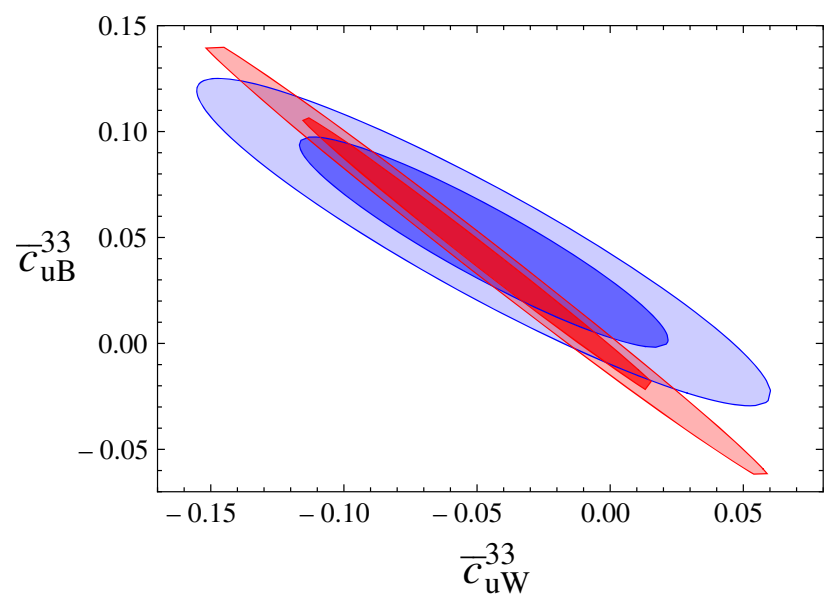

Fig. 9 Contour of the $S, T, U$ fit reported in [53] for specifically the operators $\mathcal{O}_{u W}, \mathcal{O}_{u B}$, which are unconstrained by down-sector measurements. All other Wilson coefficients are chosen to be zero. The dark and light shaded areas represent 68 and $95 \%$ confidence levels for this projections, while the blue contour uses $\mu_{R}=m_{Z}$ and the red contour $\mu_{R}=1 \mathrm{TeV}$

modifications of the gauge boson two-point functions. The operators considered in this work modify these at the oneloop level through diagrams of the type shown in Fig. 8. Throughout we perform our calculation in dimensional regularisation.

The definitions of $S, T, U$, see $[54,55,58]$, are such that in the SM all divergencies cancel when replacing the renormalised polarisation functions by their bare counterparts. The modifications of Fig. 8, however, induce additional divergencies due to the dimension 6 parts and the introduction of twopoint function counterterms is essential to obtain a UV-finite result; see also [52]. This leads to a regularisation scale $\mu_{R}$ dependence of the dimension 6 amplitude parts after renormalisation. It is this part which we focus on as we choose the SM with a $125 \mathrm{GeV}$ Higgs as reference point [53].

As can be seen from Fig. 9, oblique corrections constrain the range of the considered parameter (setting all other contributions to zero). Given that we deal with an effective theory, there is a dependence on the UV cut-off $\mu_{R}$ and constraints can be sharpened by requiring that cut-off to lie well above the $\mathrm{TeV}$ scale. As such, the oblique corrections are explicitly sensitive to concrete UV completions, and care needs to be taken not to over-emphasise their impact.

\subsection{Non-oblique corrections}

A well-measured quantity at LEP is the $Z b \bar{b}$ vertex, which enters the prediction of the bottom forward-backward asymmetry $A_{F B}^{b \bar{b}}$; see e.g. [59]. Similar to the operators in Eq. (II.4), in the generic dimension six approach we can expect similar operators for the down-sector of the third fermion family. These will modify the interactions along the same lines as we focussed on above for the top sector. However, due to the different isospin properties, the bottom forward backward asymmetry is now sensitive to the $\operatorname{sum} c_{\varphi q}^{(3)}+c_{\varphi q}^{(1)}$. This leads

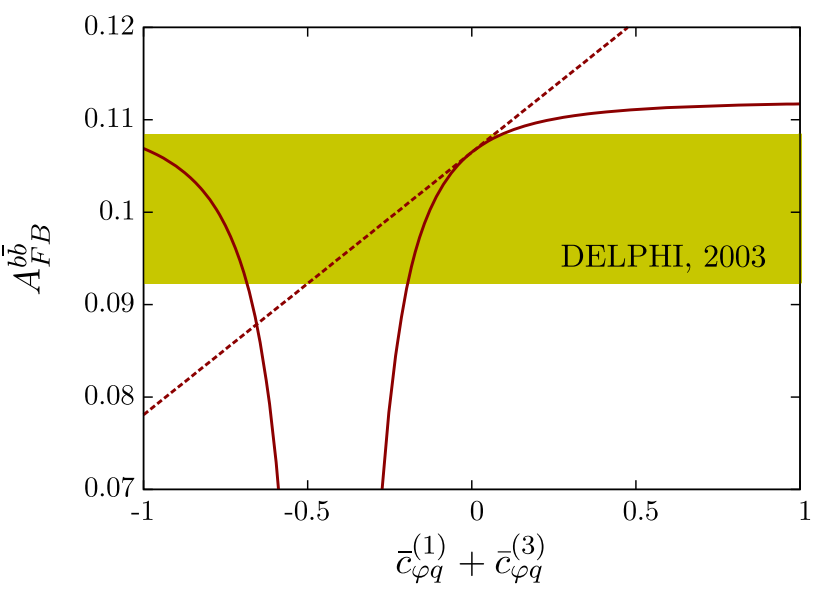

Fig. 10 Forward-backward asymmetry (linearised, dashed, and full results, solid) as a function of $c_{\varphi q}^{(3)}+c_{\varphi q}^{(1)}$. The exclusion contour is taken from DELPHI collaboration's Ref. [59] for the most constraining measurement at $\sqrt{s}=91.26 \mathrm{GeV}$

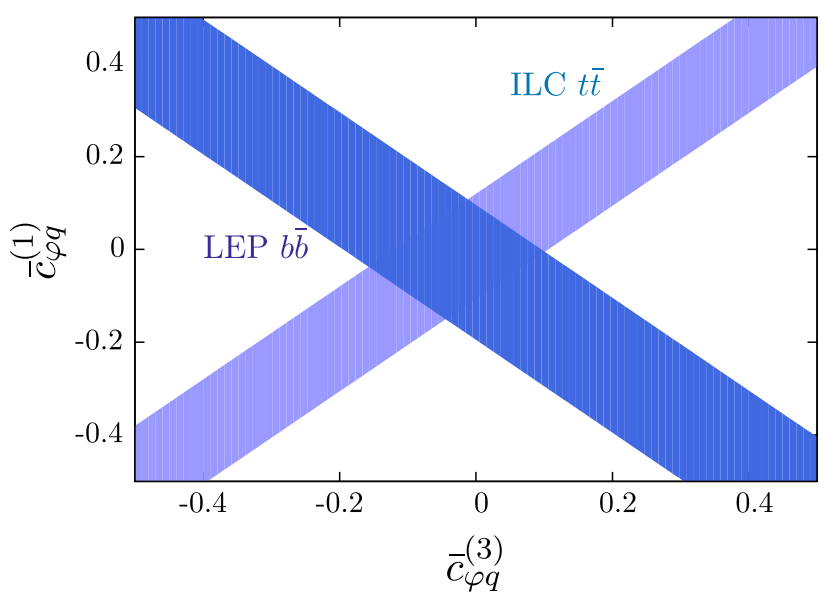

Fig. 11 Allowed 95\% confidence regions for the Wilson coefficients $\bar{c}_{\varphi u}^{(3)}$ and $\bar{c}_{\varphi u}^{(1)}$ obtained from combining the information from ILC $t \bar{t}$ asymmetries and cross-sections (dark blue) and LEP1 b $\bar{b}$ measurements (lighter blue) 
to a complementary constraint by the LEP forward backward asymmetry compared to the direct measurements in $t \bar{t}$, Fig. 10.

Moreover, the constraints on $c_{\varphi q}^{(3)}+c_{\varphi q}^{(1)}$ from $A_{F B}^{b \bar{b}}$ can be combined with the constraints on $c_{\varphi q}^{(3)}-c_{\varphi q}^{(1)}$ to extract independent bounds on $c_{\varphi q}^{(3)}$ and $c_{\varphi q}^{(1)}$. This is shown in Fig. 11. Care should be taken when interpreting these constraints individually, however. We are considering marginalised bounds for the ILC constraint but only one operator combination for the LEP bound. In general, other operators that we do not consider here will impact the $Z b \bar{b}$ vertex at tree level and in general weaken the bound. This serves as a useful visualisation, however, of the complementarity between past and future colliders in constraining these operators.

\section{Summary and conclusions}

Given the unsatisfactory precision of current probes of top quark electroweak couplings from hadron collider measurements, they must be a key priority in the physics agenda of any future linear $e^{+} e^{-}$collider. By parameterising non-standard top couplings through $D=6$ operators, we have analysed the potential for the ILC and CLIC to improve the current precision of the top electroweak sector. Unsurprisingly, if experimental precision would match current estimates, and theory uncertainties can be brought to the same level, the current constraints can be drastically improved by both colliders, with associated bounds on the scale of new physics typically in the $1 \mathrm{TeV}$ to few $\mathrm{TeV}$ range, depending on the assumed coupling structures of the underlying model. Using asymmetry measurements as well as cross-sections will be crucial to this endeavour, as will collecting large datasets with several incoming beam polarisations.

We have found that, unlike for the Higgs sector, the large increase in centre-of-mass energy at CLIC does not necessarily offer a competitive advantage over the ILC for bounding new top interactions by the operators we consider, and bounds on the operators that we consider are typically stronger at the latter, though in simultaneous 4D fits the difference is not striking. For some of the operators we consider, the bounds derived from CLIC fits correspond to mass scales smaller than the CM energy that we consider, which can call into question the validity of the EFT description, unless the CLIC sensitivity can exceed the expectations we forecast here.

By combining $Z$-pole measurements from LEP1 with $t \bar{t}$ measurements (and future improved electroweak precision measurements), one can in principle break degeneracies in the operator set and disentangle individual operators that could previously be only bounded in combinations. We showed this for the LEP forward-backward asymmetry, this could be improved by fitting other precision elec- troweak observables too. Care must be taken in interpreting the associated constraints, however, as both sets of measurements will in general talk to other operators for which there is no complementarity, and a more systematic approach taking into account EFT loop corrections would have be undertaken before these numerical bounds can be taken at face value.

Acknowledgements MR is supported in part by the UK Science and Technology Facilities Council (STFC) under Grant ST/L000446/1.

Open Access This article is distributed under the terms of the Creative Commons Attribution 4.0 International License (http://creativecomm ons.org/licenses/by/4.0/), which permits unrestricted use, distribution, and reproduction in any medium, provided you give appropriate credit to the original author(s) and the source, provide a link to the Creative Commons license, and indicate if changes were made.

Funded by $\mathrm{SCOAP}^{3}$.

\section{References}

1. H. Georgi, L. Kaplan, D. Morin, A. Schenk, Phys. Rev. D 51, 3888 (1995). arXiv:hep-ph/9410307

2. R. Contino, Y. Nomura, A. Pomarol, Nucl. Phys. B 671, 148 (2003). arXiv:hep-ph/0306259

3. K. Agashe, R. Contino, A. Pomarol, Nucl. Phys. B 719, 165 (2005). arXiv:hep-ph/0412089

4. H.P. Nilles, Phys. Rep. 110, 1 (1984)

5. R. Contino, A. Falkowski, F. Goertz, C. Grojean, F. Riva, JHEP 07, 144 (2016). arXiv: 1604.06444

6. A. Buckley, C. Englert, J. Ferrando, D.J. Miller, L. Moore, M. Russell, C.D. White, Phys. Rev. D 92, 091501 (2015). arXiv: 1506.08845

7. A. Buckley, C. Englert, J. Ferrando, D.J. Miller, L. Moore, M. Russell, C.D. White, JHEP 04, 015 (2016). arXiv: 1512.03360

8. O. Bessidskaia Bylund, F. Maltoni, I. Tsinikos, E. Vryonidou, C. Zhang, JHEP 05, 052 (2016). arXiv: 1601.08193

9. G. Durieux, F. Maltoni, C. Zhang, Phys. Rev. D 91, 074017 (2015). arXiv: 1412.7166

10. R. Röntsch, M. Schulze, JHEP 07, 091 (2014). arXiv:1404.1005 [Erratum: JHEP09,132(2015)]

11. M.P. Rosello, M. Vos, Eur. Phys. J. C 76, 200 (2016). arXiv: 1512.07542

12. M. Schulze, Y. Soreq, Eur. Phys. J. C 76, 466 (2016) arXiv: 1603.08911

13. J. de Blas, M. Chala, J. Santiago, JHEP 09, 189 (2015). arXiv: 1507.00757

14. J.A. Aguilar-Saavedra, J. Carvalho, N.F. Castro, F. Veloso, A. Onofre, Eur. Phys. J. C 50, 519 (2007). arXiv:hep-ph/0605190

15. C. Englert, M. Spannowsky, Phys. Lett. B 740, 8 (2015). arXiv: 1408.5147

16. Y. Kiyo, A. Maier, P. Maierhofer, P. Marquard, Nucl. Phys. B 823, 269 (2009). arXiv:0907.2120

17. L. Chen, O. Dekkers, D. Heisler, W. Bernreuther, Z.-G. Si, JHEP 12, 098 (2016). arXiv: 1610.07897

18. M. Klute, R. Lafaye, T. Plehn, M. Rauch, D. Zerwas, Europhys. Lett. 101, 51001 (2013). arXiv:1301.1322

19. H. Khanpour, M. Mohammadi Najafabadi, Phys. Rev. D 95, 055026 (2017). arXiv: 1702.00951

20. M. Vos (2017). arXiv:1701.06537

21. M. Vos et al. (2016). arXiv:1604.08122

22. M.S. Amjad et al., Eur. Phys. J. C 75, 512 (2015). arXiv: 1505.06020

23. R. Röntsch, M. Schulze, JHEP 08, 044 (2015). arXiv:1501.05939 
24. B. Coleppa, M. Kumar, S. Kumar, B. Mellado. (2017). arXiv: 1702.03426

25. Q.-H. Cao, B. Yan, Phys. Rev. D 92, 094018 (2015). arXiv:1507.06204

26. B. Grzadkowski, M. Iskrzynski, M. Misiak, J. Rosiek, JHEP 10, 085 (2010). arXiv: 1008.4884

27. V. Cirigliano, W. Dekens, J. de Vries, E. Mereghetti, Phys. Rev. D 94, 016002 (2016). arXiv:1603.03049

28. A. Alloul, N.D. Christensen, C. Degrande, C. Duhr, B. Fuks, Comput. Phys. Commun. 185, 2250 (2014). arXiv:1310.1921

29. C. Degrande, C. Duhr, B. Fuks, D. Grellscheid, O. Mattelaer, T. Reiter, Comput. Phys. Commun. 183, 1201 (2012). arXiv:1108.2040

30. J. Alwall, R. Frederix, S. Frixione, V. Hirschi, F. Maltoni, O. Mattelaer, H.S. Shao, T. Stelzer, P. Torrielli, M. Zaro, JHEP 07, 079 (2014). arXiv: 1405.0301

31. T. Hahn, Comput. Phys. Commun. 140, 418 (2001). arXiv:hep-ph/0012260

32. T. Hahn, M. Perez-Victoria, Comput. Phys. Commun. 118, 153 (1999). arXiv:hep-ph/9807565

33. F. James, M. Roos, Comput. Phys. Commun. 10, 343 (1975)

34. A. Buckley, H. Hoeth, H. Lacker, H. Schulz, J.E. von Seggern, Eur. Phys. J. C 65, 331 (2010). arXiv:0907.2973

35. F. Maltoni, D. Pagani, I. Tsinikos, JHEP 02, 113 (2016). arXiv: 1507.05640

36. M. Aaboud et al. (collaboration ATLAS), Eur. Phys. J. C 77, 40 (2017). arXiv:1609.01599

37. Tech. Rep. CMS-PAS-TOP-16-017, CERN, Geneva (2016). https://cds.cern.ch/record/2205283

38. C. Englert, L. Moore, K. Nordström, M. Russell, Phys. Lett. B 763, 9 (2016). arXiv: 1607.04304

39. S.T. Boogert, D.J. Miller, in Linear colliders. Proceedings, International Workshop on physics and experiments with future electron-positron linear colliders, LCWS 2002, Seogwipo, Jeju Island, Korea, August 26-30, 2002 (2002), pp. 509516, arXiv:hep-ex/0211021. http://alice.cern.ch/format/showfull? sysnb $=2349913$
40. B. Chokoufé Nejad, W. Kilian, J.M. Lindert, S. Pozzorini, J. Reuter, C. Weiss, JHEP 12, 075 (2016). arXiv:1609.03390

41. J. Fleischer, A. Leike, T. Riemann, A. Werthenbach, Eur. Phys. J. C 31, 37 (2003). arXiv:hep-ph/0302259

42. J. Gao, H.X. Zhu, Phys. Rev. D 90, 114022 (2014a). arXiv: 1408.5150

43. W. Bernreuther, R. Bonciani, T. Gehrmann, R. Heinesch, T. Leineweber, P. Mastrolia, E. Remiddi, Nucl. Phys. B 750, 83 (2006). arXiv:hep-ph/0604031

44. J. Gao, H.X. Zhu, Phys. Rev. Lett. 113, 262001 (2014b). arXiv: 1410.3165

45. K.-I. Hikasa, Phys. Rev. D 33, 3203 (1986)

46. G. Moortgat-Pick et al., Phys. Rep. 460, 131 (2008). arXiv:hep-ph/0507011

47. M.S. Amjad, M. Boronat, T. Frisson, I. Garcia, R. Poschl, E. Ros, F. Richard, J. Rouene, P.R. Femenia, M. Vos. (2013). arXiv: 1307.8102

48. M.J. Boland et al. (collaboration CLICdp, CLIC). (2016). arXiv: 1608.07537

49. H. Abramowicz et al. (2016). arXiv: 1608.07538

50. J. Ellis, P. Roloff, V. Sanz, T. You. (2017). arXiv:1701.04804

51. L. Berthier, M. Trott, JHEP 05, 024 (2015). arXiv: 1502.02570

52. M. Ghezzi, R. Gomez-Ambrosio, G. Passarino, S. Uccirati, JHEP 07, 175 (2015). arXiv: 1505.03706

53. M. Baak, J. Cuth, J. Haller, A. Hoecker, R. Kogler, K. Mönig, M. Schott, J. Stelzer (collaboration Gfitter Group), Eur. Phys. J. C 74, 3046 (2014). arXiv: 1407.3792

54. M.E. Peskin, T. Takeuchi, Phys. Rev. Lett. 65, 964 (1990)

55. M.E. Peskin, T. Takeuchi, Phys. Rev. D 46, 381 (1992)

56. D.A. Ross, M.J.G. Veltman, Nucl. Phys. B 95, 135 (1975)

57. C. Grojean, E.E. Jenkins, A.V. Manohar, M. Trott, JHEP 04, 016 (2013). arXiv: 1301.2588

58. R. Barbieri, A. Pomarol, R. Rattazzi, A. Strumia, Nucl. Phys. B 703, 127 (2004). arXiv:hep-ph/0405040

59. J. Abdallah et al. (collaboration DELPHI), Eur. Phys. J. C 34, 109 (2004). arXiv:hep-ex/0403041 